\title{
ESTUDIO SOBRE INTELIGENCIA EMOCIONAL, SATISFACCIÓN VITAL Y OPTIMISMO DISPOSICIONAL EN UN CENTRO DE DÍA OCUPACIONAL DE PERSONAS CON DISCAPACIDAD INTELECTUAL
}

\section{A study about Emotional Intelligence, vital satisfaction and dispositional optimism in an ocupational day center of people with intellectual disability}

Óscar Gavín ChOcano

Universidad de Jaén. Departamento de Investigación y Diagnóstico en Educación (MIDE) del Departamento de Pedagogía

oscar_gavin@yahoo.es

David Molero

Universidad de Jaén. Departamento de Investigación y Diagnóstico en Educación (MIDE) del Departamento de Pedagogía

Recepción: 14 de noviembre de 2018

Aceptación definitiva: 4 de febrero de 2019

Resumen: El propósito de este estudio es aportar evidencias sobre la relación de la inteligencia emocional, satisfacción vital y optimismo disposicional en las personas con discapacidad intelectual que asisten a un centro de día y terapia ocupacional. La muestra está compuesta por 15 sujetos $(n=15)$, con una edad media de 34.93 años; DT $( \pm 12.28)$ y un grado de discapacidad promedio del $64.07 \%$. Para la obtención de información se utilizan como instrumentos: Trait Meta-Mood Scale-24 (TMMS-24), Emotional Quotient inventory (EQi-C), Satisfaction with Life Scale (SWLS) y Life Orientation Test Revised (LOT-R). Las evidencias muestran que los participantes son capaces de mostrar 
una conducta adaptativa a circunstancias adversas y, por tanto, alcanzar un alto nivel de satisfacción vital y optimismo. Existen relaciones entre algunas de las dimensiones de los instrumentos considerados $(p<.05)$ y hay diferencias significativas a nivel estadístico entre las dimensiones de inteligencia emocional y las variables sociodemográficas grado de discapacidad y edad $(p<.05)$. El análisis de regresión confirma la asociación positiva entre inteligencia emocional (adaptabilidad) con la satisfacción vital. Se aborda una discusión de los resultados con investigaciones realizadas en colectivos con las mismas características, concluyendo la falta de estudios que aborden inteligencia emocional, satisfacción con la vida y optimismo disposicional en colectivos con discapacidad.

Palabras Clave: discapacidad intelectual; inteligencia emocional percibida; satisfacción vital; optimismo disposicional.

AвSTRACT: The purpose of this study is to provide evidence about the relation between emotional intelligence, vital satisfaction and dispositional optimism in mentally disabled people that attend a daily and occupational therapy centre. The sample is composed of 15 individuals $(n=15)$, of an average age of 34.93 years old; DT ( \pm 12.28$)$ and an average disability degree of $64.07 \%$. In order to obtain the information, some measurement instruments have been used: Trait Meta-Mood Scale-24 (TMMS-24), Emotional Quotient inventory (EQi-C), Satisfaction with Life Scale (sWLS) and Life Orientation Test Revised (LOT-R). The evidences show that the participants are able to show an adaptive behaviour against adverse circumstances and as a consequence being able to achieve a high level of vital fulfillment and optimism. There are relations between the dimensions of the considered instruments $(p<.05)$ and there are also significative differences on an statistic level in the emotional intelligence dimension as well as in the social-demographic variables of disability degree and age $(p<.05)$. The regression test confirms the positive association between emotional intelligence (adaptability) and vital satisfaction. It tackles a discussion about the results of the test done on groups with similar characteristics, concluding that there is a lack of studies made that tackle the emotional intelligence, life fulfilment and dispositional optimism on groups of disabled individuals.

KEY WORDS: intellectual disability; perceived emotional intelligence; vital satisfaction; dispositional optimism.

\section{Introducción}

L OS OBjETIVOS Y LOgROS SUbyACENTES al denominado Estado del Bienestar durante la segunda mitad del siglo xx constituyeron la base de nuevas políticas sociales, propósito inherente a la conceptualización y desarrollo de la Inteligencia Emocional (en adelante IE), vinculada al bienestar y satisfacción del individuo, así como con el optimismo y el pesimismo disposicional en relación a su contexto. Este estudio se centra en la importancia del análisis de estas variables en personas con discapacidad intelectual (en adelante DI).

Dichos logros, relacionados con el bienestar, justicia social y protección de colectivos menos favorecidos, fueron recorriendo un camino paralelo con el modelo de

(C) Ediciones Universidad de Salamanca / CC BY-NC-ND

Siglo Cero, vol. 50 (3), n. ${ }^{\circ}$ 271, 2019, julio-septiembre, pp. 47-65 
IE desarrollado por Salovey y Mayer (1990), formalizando una idea basada en el uso adaptativo de los procesos cognitivos y emociones, que permitiera al individuo un funcionamiento adecuado en conexión con el contexto (Cazalla-Luna y Molero, 2016), ayudando a conocer otras cualidades personales (Alonso-Ferres, Berrocal de Luna y Jiménez-Sánchez, 2018), y que a su vez dotara al mismo de herramientas adecuadas capaces de solucionar problemas (Fernández-Berrocal y Ruiz-Aranda, 2008), pudiendo evaluar su proceso a lo largo de la vida con nuevos instrumentos (Fernández-Berrocal, Ruiz-Aranda, Salguero, Palomera y Extremera, 2017). El interés por esta temática es creciente, y se han aportado criterios que han servido de nexo a las diferentes conceptualizaciones de la IE, apareciendo dos modelos claramente diferenciados: "modelo IE habilidad" y "modelo mixto o rasgo" (Mayer, Roberts y Barsade, 2008).

Si nos centramos en el primero, "modelo habilidad", considerando la IE como la capacidad de percibir, comprender y manejar las emociones (Mayer, Salovey y Caruso, 2000), conceptualizando la IE a través de cuatro habilidades básicas: percepción, comprensión, facilitación y regulación emocional (Cabello, Ruiz-Aranda y Fernández-Berrocal, 2009).

Por otro lado, el "modelo rasgo", o también llamado "modelo mixto", combina las dimensiones de personalidad con habilidades emocionales (Mayer et al., 2008). Entiende la IE como un conjunto de comportamientos autopercibidos relacionados con las propias capacidades, utilizando esa información en el contexto (Petrides y Furham, 2001), incluyendo en la misma conceptos como empatía, asertividad, satisfacción vital y optimismo.

Estos dos modelos comparten conceptos básicos en cuanto a su base estructural y núcleo, dotando a estudios sobre IE de una nueva perspectiva en el análisis de las emociones (MacCann, Joseph, Newman y Roberts, 2014). Es decir, la personas con mayor IE tendrán la capacidad de entender las emociones de otras personas, aportando mayores recursos para discriminar comportamientos negativos (López-Cassa, Pérez-Escoda y Alegre, 2018).

Los aspectos analizados permiten implementar un desarrollo en las capacidades emocionales, incrementan la posibilidad de afrontar situaciones adversas, mejorando la satisfacción vital (Pulido y Herrera, 2018) desde diferentes enfoques (emocional o afectivo y cognitivo). Un enfoque emocional o afectivo puede producir equilibrio, el cual viene determinado por afectos positivos y negativos (Bradburn, 1969). Un enfoque cognitivo, centrado en la forma como los individuos evalúan su vida, comparándolo con su ideal de vida (Liberalesso, 2002) y con mayor consistencia transituacional y estabilidad temporal que la felicidad (Bassi, Bacher, Negri y Delle Fave, 2013).

Los conceptos descritos con anterioridad están ligados a la satisfacción con la vida, bienestar autopercibido y la prioridad de los sentimientos positivos sobre los negativos (Veloso-Besio, Cuadra-Peralta, Antezana-Saguez, Avendaño-Robledo y Fuentes-Soto, 2013), conceptualizada como reflexión personal y global sobre la propia vida y relación al contexto próximo (Cazalla-Luna y Molero, 2018). Numerosos investigadores encuentran útil esta distinción en la comprensión de bienestar autopercibido (Veloso-Besio et al., 2013), entendiendo que las personas con un alto grado de bienestar percibido y satisfacción vital podrán fundamentar su vida de una manera 
óptima (Cazalla-Luna y Molero, 2018). De igual forma son numerosas las investigaciones que relacionan IE, bienestar percibido y satisfacción vital (Andrei y Petrides, 2013; Cejudo, López-Delgado y Rubio, 2016; Extremera y Fernández-Berrocal, 2006; Extremera, Salguero y Fernández-Berrocal, 2011; Fernández-Abascal y Martín-Díaz, 2015; Mavroveli, Petrides, Sangareau y Furnham, 2009; Petrides, Pérez-González y Furnham, 2007; Thompson, Waltz, Croyle y Pepper, 2007).

En los últimos años, algunos autores relacionan positivamente satisfacción con la vida, en su aspecto cognitivo y afectivo, con otros conceptos como esperanza de vida, apoyo social percibido, optimismo disposicional y salud (Diener, Oishi y Ryan, 2013; Rodríguez, 2015; Rojas y Veenhoven, 2013; Schimmack, 2007), entendiendo este último como la disposición del individuo a evaluar de forma positiva la concurrencia y proyección a futuros eventos, no siendo necesaria la situación actual de su vida (Carver, Scheier y Segerstrom, 2010; Gázquez-Linares, Pérez-Fuentes, Mercader-Rubio e Inglés-Saura, 2014; Pedrosa, Celis-Atenas, Suárez-Álvarez, García-Cueto y Muñiz, 2015). Aunque exista cierta disparidad respecto a la doble dimensión del constructo, encontrando diferentes trabajos que interpretan que un individuo podría presentar expectativas optimistas en general y una perspectiva pesimista en una situación específica (Vera-Villarroel, Pávez y Silva, 2012). En este sentido, algunos trabajos proponen tratar optimismo y pesimismo como una única dimensión (Carver y Scheier, 2014).

Recientemente se ha relacionado satisfacción con la vida con optimismo disposicional (Avey, Luthans, Smith y Palmer, 2010; Chavarria y Barra, 2014; Contreras, Lillo y Vera-Villarroel, 2015; Fernandes de Araújo, Teva y Bermúdez, 2015; LondoñoPérez, Velasco-Salamanca, Alejo-Castañeda, Botero-Soto y Vanegas, 2014; Marrero y Carballeira, 2010; Novoa y Barra, 2015; Von Humboldt y Leal, 2014; Xu y Roberts, 2010), no siendo concluyentes los resultados obtenidos al no permitir determinar el nivel de bienestar (Fernández-Berrocal et al., 2017).

Propuestas como las de Vera-Villarroel et al. (2012) sobre el modelo explicativo del bienestar postulan el optimismo como una posible variable que predispone a un mayor bienestar, sustentando la conexión positiva entre estas dos variables (AugustoLanda, Pulido-Martos y López-Zafra, 2011); sin embargo, estudios sobre variables disposicionales cuestionan esta afirmación, al considerar que esta relación estaría sustentada por los afectos (Schimmack, 2007; Şimşek y Koydemir, 2013) o por el grado de competencias emocionales adquiridas que determinan la IE.

Otras aportaciones subrayan la relación entre IE, satisfacción con la vida y optimismo disposicional en personas con DI, pero aún son escasas, siendo de mayor proliferación artículos relacionados con padres y madres de hijos con DI (Manor-Binyamini y Nator, 2016) y profesionales de centros de personas con Di (Bazzano, Wolfe, Zylowska, Wang, Schuster, Barrett y Lehrer, 2015; Extremera, Duran y Rey, 2005).

Teniendo en cuenta los antecedentes y argumentos previos, los objetivos considerados en este estudio, con carácter general, son: (a) Analizar las puntuaciones obtenidas en IE, satisfacción vital y optimismo disposicional en los participantes; $(b)$ Determinar la existencia de correlaciones significativas en las dimensiones de los instrumentos de evaluación de la IE Trait Meta-Mood Scale-24 (TMMS-24), Emotional 
Quotient inventory (EQi-C), satisfacción vital, Satisfaction with Life Scale (swLS) y optimismo disposicional, Life Orientation Test Revised (LOT-R); (c) Establecer la existencia de diferencias significativas entre las dimensiones de los instrumentos considerados (EQi-C, TMMS-24, SWLS y LOT-R) y las variables sociodemográficas (género, grado de discapacidad y edad y (d) Predecir qué variables de la IE y del optimismo son las que más explican la satisfacción con la vida de los sujetos de la muestra.

\section{Método}

El diseño del presente estudio está basado fundamentalmente en una investigación de tipo empírico, no experimental, empleando estrategias de investigación asociativa y descriptiva (Ato, López y Benavente, 2013). A partir de los criterios establecidos, se han desarrollado medidas de carácter transversal, empleando estrategias comparativas y de predicción.

\subsection{Participantes}

Considerando el tamaño reducido de la muestra y la complejidad asumida para la obtención de información a un mayor número de sujetos, partimos de la oportunidad para profundizar en un contexto concreto y características especiales, siendo los hallazgos obtenidos contrastables con grupos más amplios en futuras investigaciones.

Siendo la totalidad de integrantes del Centro de Día y Terapia Ocupacional $(n=$ 20) de la Ciudad de Linares (Jaén, España), se consideró fundamental para el estudio descartar aquellos sujetos, que debido al grado de DI no pudiesen realizar las diferentes pruebas, con o sin los apoyos necesarios, siendo la muestra resultantes de $(n=15)$, 8 mujeres $(53.5 \%)$ y 7 hombres $(46.65 \%)$, con un grado de discapacidad media del $64.07 \%$; DT $( \pm 9.83)$. El intervalo en la edad oscila entre 17 y 63 años, con una media de 34.93; DT ( \pm 12.28$)$. Hemos empleado un muestreo no probabilístico de tipo causal o accidental (Latorre, Del Rincón y Arnal, 2003).

\subsection{Instrumentos}

Variables sociodemográficas. Se han incluido tres variables sociodemográficas: género, grado de discapacidad y edad, con el propósito de obtener información de la muestra y determinar la existencia de diferencias significativas en función de estas.

Trait Meta-Mood Scale-24-. Para evaluar la IE se utilizó la escala Trait Meta-Mood Scale-24 (TMms-24), de Fernández-Berrocal, Extremera y Ramos (2004), versión original de Salovey, Mayer, Goldman, Turvey y Palfai (1995), que engloba tres dimensiones (atención, claridad y reparación emocional). Esta herramienta ha sido utilizada en multitud de contextos de investigación en Ciencias Sociales (Tolegenova, Kustubayeva y Matthews, 2014) y de las Ciencias de la Salud (Aradilla-Herrero, Tomás- 
Sábado y Gómez-Benito, 2014; Lara, Carranque, Oehling, Hernández y Oliva, 2014; Munkholm, Vinberg, Berk \& Kessing, 2012). Consta de una consistencia interna establecida por sus autores de $\alpha=.90$ para atención, $\alpha=.90$ en claridad y $\alpha=.86$ para reparación de las emociones. En nuestra muestra la fiabilidad de las puntuaciones para cada subescala es de $\alpha=.86, \alpha=.59$ y $\alpha=.91$, respectivamente.

Emotional Quotient Inventory. Para evaluar la IE se utilizó la Emotional Quotient Inventory -EQi-C- (López-Zafra et al., 2014). Es una adaptación al español (versión corta) del EQ-i (Bar-On, 1997) y su versión para jóvenes y adolescentes EQ-i: YV (BarOn y Parker, 2000). Ofrece información relativa a competencias emocionales y su relación con otras variables, contando con cuatro factores (interpersonal, adaptabilidad, manejo del estrés e intrapersonal) para el estudio de la IE, siendo los valores de la consistencia interna informados por sus autores de $\alpha=.78, \alpha=.75, \alpha=.73$ y $\alpha=.70$ para cada subescala. En nuestra muestra la fiabilidad de las puntuaciones para cada subescala del EQi-C es de $\alpha=.85, \alpha=0.78, \alpha=.96$ y $\alpha=.77$, respectivamente.

Satisfaction with Life Scale. Para evaluar la satisfacción vital, se utilizó la Satisfaction with Life Scale -SWLS- (Diener, Emmons, Larsen y Griffin, 1985). Compuesta por cinco sencillos ítems donde los participantes deben indicar el grado de acuerdo o desacuerdo con las opciones de respuesta del instrumento. La escala en la versión española establece una consistencia interna de $\alpha=.82$. La fiabilidad de las puntuaciones en nuestra muestra es $\alpha=.93$.

Life Orientation Test Revised. Para evaluar el optimismo disposicional se utilizó la versión en español del Life Orientation Test Revised-LOT-R-(Remor, Amorós y Carrobles, 2006). Escala compuesta por diez ítems que miden el grado de optimismo de los sujetos. El formato de respuesta responde a una escala de tipo Likert de cinco puntos. Se estima que a mayor valoración mayor optimismo disposicional. La consistencia interna informada por sus autores es de $\alpha=.78$; siendo la fiabilidad de las puntuaciones en nuestro estudio de $\alpha=.81$.

\subsection{Procedimiento}

El estudio se llevó a cabo en las instalaciones de la entidad, previa autorización de los responsables, obteniendo consentimiento informado de cada participante, así como de familiares y tutores. Los sujetos fueron informados del proceso a seguir, confidencialidad y anonimato de las evidencias recogidas. Cada una de las pruebas se realizó de forma colectiva, prestando el apoyo necesario, teniendo en cuenta las características del colectivo. Se estimó un tiempo aproximado de 90 minutos para cada instrumento, con recesos de 5 minutos. Se contestaron los cuestionarios en siguiente orden: preguntas sociodemográficas, TMMS-24, EQi-C, SWLS y LOT-R.

\subsection{Análisis de los datos}

A partir de los datos recogidos, se obtuvieron estadísticos descriptivos (medias y desviaciones típicas), analizando después la fiabilidad de las puntuaciones (consistencia 
interna, alfa de Cronbach) y la correlación entre las puntuaciones resultantes en cada una de las dimensiones. A continuación, se realizó un análisis de diferencias de medias en función del género y grado de discapacidad (prueba $U$ de Mann-Whitney de diferencia de medias para muestras no relacionadas); para la variable edad (expresada en tres intervalos) se empleó la prueba $H$ de Kruskal-Wallis. Se han empleado pruebas no paramétricas al no cumplirse el supuesto de normalidad en todos los casos en función de los datos obtenidos en la prueba Shapiro-Wilk (empleamos esta prueba al ser $n<30$ casos); además se informa del tamaño del efecto en los análisis realizados. Finalmente, para explorar el valor predictivo de las variables IE y optimismo disposicional sobre la satisfacción vital se realizó un estudio de regresión paso a paso. En todos los casos se empleó un nivel de confianza del 95\% (significación $p<.05$ ), empleando el programa SPSS 22.0 (IBM, Chicago, IL) para obtener los resultados de las pruebas indicadas con anterioridad.

\section{Resultados}

\subsection{Relación entre satisfacción vital, optimismo disposicional e inteligencia emocional}

En primer lugar, presentamos en la Tabla 1 la matriz de correlaciones ( $r$ de Pearson) junto a los estadísticos descriptivos (medias y desviaciones típicas) y la fiabilidad de las puntuaciones (alfa de Cronbach) de las dimensiones o subescalas empleadas en el estudio (ya analizadas en el apartado de los instrumentos). Hemos encontrado

\begin{tabular}{l} 
TABLA 1. Consistencia interna, medias, desviación típica y correlaciones \\
(r de Pearson) entre las variables satisfacción vital, optimismo disposicional \\
e inteligencia emocional \\
Variable \\
\hline SV
\end{tabular}

Nota: (1) Media = M, Desviación típica $=D T$, Satisfacción vital = sv, Optimismo $=$ OPT, Inteligencia emocional Atención $=$ AT, Claridad $=$ CL, Reparación $=$ RE, Inteligencia emocional Interpersonal $=$ INT, Adaptabilidad $=$ $\mathrm{AD}$, Manejo del estrés $=\mathrm{EST}$, Intrapersonal $=$ INTR. $(2) *=p<.05 ; * *=p<.01$. 
relaciones positivas estadísticamente significativas entre las dimensiones IE adaptabilidad y satisfacción vital $\left(r_{(15)}=.53 ; p<.05\right)$, IE claridad y optimismo disposicional $\left(\mathrm{r}_{(15)}=.54 ; p<.05\right)$, IE adaptabilidad y claridad $\left(r_{(15)}=.57 ; p<.05\right)$, IE adaptabilidad y reparación $\left(r_{(15)}=.54 ; p<.05\right)$, IE adaptabilidad e interpersonal $\left(r_{(15)}=0,70 ; p<.01\right)$, IE claridad y reparación $\left(\mathrm{r}_{(15)}=.75 ; p<.01\right)$ y también entre IE intrapersonal y optimismo disposicional $\left(r_{(15)}=.52 ; p<.05\right)$. Finalmente, destacamos que la dimensión atención de la IE correlacionada significativamente con el optimismo disposicional, pero de manera inversa $\left(r_{(15)}=-.57 ; p<.05\right)$. Es posible que no hayamos encontrado correlaciones significativas entre todas las dimensiones de los dos instrumentos que valoran la IE al basarse cada uno de ellos en modelos de la IE distintos, TMMs-24, en un modelo mixto y el EQi-C en el modelo rasgo.

\subsection{Diferencias en función de las variables sociodemográficas}

Para analizar las diferencias en relación al género se ha empleado la prueba no paramétrica $U$ de Mann-Whitney para dos muestras independientes (véase Tabla 2). Los resultados indican que no existen diferencias significativas entre ninguna de las dimensiones del TMMs-24 (atención, claridad y reparación) en relación al género $(z<$ 2.0; $p>.05 \mathrm{~ns}$ ). Tampoco en las dimensiones del EQi-C (interpersonal, adaptabilidad, manejo del estrés e intrapersonal) hemos encontramos diferencias significativas en

\begin{tabular}{|c|c|c|c|c|c|}
\hline \multicolumn{7}{|c|}{ TABLA 2. Diferencias de medias en función del género } \\
(Prueba U de Mann-Whitney)
\end{tabular}

Nota: (1) Media = M, Desviación típica $=D T$, Mann-Whitney = Z, Optimismo = OPT, Inteligencia emocional Atención = IEA, Claridad = IEAT, Reparación = IERE, Inteligencia emocional Interpersonal = IEINTER, Adaptabilidad = IEADAP, Manejo del estrés = IEEST, Intrapersonal = IEINTRA. (2) El tamaño del efecto estadístico está expresado con el valor de Cohen (pequeño $=.2$, mediano $=.5$ y grande $=.8) .(3) *=p<.05$; $* *=p<.01$. 
relación al género $(z<2.0 ; p>.05 \mathrm{~ns})$. Tal y como hemos anticipado, ni en satisfacción vital $(Z=-.581 ; p>.05 \mathrm{~ns})$ ni en optimismo disposicional $(Z=-.642 ; p>.05 \mathrm{~ns})$ se ha encontrado significatividad en las diferencias. Para calcular el tamaño del efecto para esta prueba no paramétrica, obtenemos el valor de $r[\mathrm{r}=\mathrm{z} / \sqrt{ } n]$, siguiendo las recientes recomendaciones de Wasserstein y Lazar (2016). El tamaño del efecto es pequeño en todos los casos $(r<.2)$; salvo en las dimensiones IEC y IERE del TMMS-24 (.419 y .329 , respectivamente), así como en la dimensión IEINTRA del EQi-C (.242), donde es de tamaño mediano según los criterios de Cohen (1988).

Para analizar las diferencias en relación al grado de discapacidad (-66\% vs. $+66 \%$ ) se empleó la prueba no paramétrica $U$ de Mann-Whitney para dos muestras independientes (véase Tabla 3). Los resultados indican que no existen diferencias significativas en satisfacción vital con respecto a la variable grado de discapacidad $(z<2.0 ; p>.05$ $n s)$. Tampoco existen diferencias significativas entre optimismo disposicional y grado de discapacidad ( $z<2.0 ; p>.05 \mathrm{~ns}$ ). En relación a las dimensiones de IE TMMS-24 (atención, claridad y reparación) no encontramos diferencias significativas en relación al grado de discapacidad $(z<2.0 ; p>.05 \mathrm{~ns}$ ) y el tamaño del efecto es mediano.

Tan solo encontramos diferencias significativas en las dimensiones del EQi-C interpersonal $(z=-2.390 ; p=.017)$, siendo esta favorable al grupo que posee menor grado de discapacidad, como era previsible. Es relevante destacar que el tamaño del efecto es mediano en todas las dimensiones de este instrumento $(.617, .480, .269$ y .385 ,

\begin{tabular}{|c|c|c|c|c|c|}
\hline \multicolumn{7}{|c|}{ TABLA 3. Diferencias de medias en función del grado de discapacidad } \\
(Prueba U de Mann-Whitney)
\end{tabular}

Nota: (1) Media = M, Desviación típica $=D T$, Mann-Whitney $=Z$, Optimismo $=$ OPT, Inteligencia emocional Atención = IEA, Claridad = IEAT, Reparación = IERE, Inteligencia emocional Interpersonal = IEINTER, Adaptabilidad = IEADAP, Manejo del estrés = IEEST, Intrapersonal = IEINTRA. (2) El tamaño del efecto estadístico está expresado con el valor de Cohen (pequeño $=.2$, mediano $=.5$ y grande $=.8) .(3) *=p<.05$; $* *=p<.01$. 
respectivamente). No encontramos diferencias significativas en las restantes subescalas del EQi-C (adaptabilidad, manejo del estrés e intrapersonal) respecto al grado de discapacidad $(z<2.0 ; p>.05 \mathrm{~ns})$.

Para analizar las diferencias en función de la edad se determinaron tres intervalos (-25 años, 26-39 años y +40 años), realizándose la prueba no paramétrica $H$ de Krus$k a l$ Wallis (véase Tabla 4). En las dimensiones satisfacción vital $\left(\chi^{2}=2.644 ; p>.05\right.$ $\mathrm{ns})$ y optimismo disposicional $\left(\chi^{2}=3.552 ; p>.05 \mathrm{~ns}\right)$, no se encontraron diferencias significativas en relación a la edad. En la variable IE TMMS-24 (atención) tampoco se encontraron diferencias significativas en relación a la edad $\left(\chi^{2}=2.382 ; p>.05 \mathrm{~ns}\right)$.

Sí se encontraron diferencias significativas en IE TMMS-24 (claridad y reparación) en relación a la edad $\left(\chi^{2}=6.020 ; p=.049\right)$ y $\left(\chi^{2}=7.259 ; p=.027\right)$ respectivamente. Las comparaciones a posteriori informan de diferencias significativas entre los participantes de menos de 25 años y los correspondientes al intervalo 26 a 39 años, favorable a los de más edad, siendo el tamaño del efecto, Épsilon al cuadrado $\left(E^{2}\right)$, mediano $\left(E^{2}=\right.$ .430 y $\left.E^{2}=.518\right)$.

En las variables IE EQi-C (interpersonal, manejo del estrés e intrapersonal) tampoco se encontraron diferencias significativas en relación a la edad $\left(\chi^{2}=4.125 ; p>.05\right.$ $\mathrm{ns}),\left(\chi^{2}=.498 ; p>.05 \mathrm{~ns}\right)$ y $\left(\chi^{2}=1.158 ; p>.05 \mathrm{~ns}\right)$, encontrando diferencias significativas en IE EQi-C (adaptabilidad) y la edad $\left(\chi^{2}=6.504 ; p=.039\right)$. La comparación a posteriori realizada informa de que la significatividad se da entre el intervalo 26 a 39 años y mayores de 40 años en IE EQi-C (adaptabilidad), siendo favorables a los sujetos del intervalo de 26 a 39 años, informando de un tamaño del efecto mediano $\left(E^{2}=.464\right)$.

\begin{tabular}{|c|c|c|c|c|c|c|}
\hline \multicolumn{7}{|c|}{$\begin{array}{l}\text { Tabla 4. Diferencias de medias en función de la edad } \\
\text { (Prueba H de Kruskal Wallis) }\end{array}$} \\
\hline Variable & $\begin{array}{c}<25 \text { años } \\
M(D T)\end{array}$ & $\begin{array}{c}\text { 26-39 años } \\
M(D T)\end{array}$ & $\begin{array}{c}>+40 \text { años } \\
M(D T)\end{array}$ & $\chi^{2}$ & $p$ & Efecto $\left(E^{2}\right)$ \\
\hline SV & $9.00(.00)$ & $14.71(5.99)$ & $8.20(2.58)$ & 2.644 & .267 & .188 \\
\hline OPT & $7.67(6.65)$ & $15.57(3.95)$ & $13.40(5.12)$ & 3.552 & .169 & .253 \\
\hline & & & $2080=238$ & 2382 & 304 & 170 \\
\hline IEC & $17.67(3.78)$ & $23.57(3.50)$ & $19.20(3.63)$ & 6.020 & $.049 *$ & .430 \\
\hline IERE & $18.33(8.38)$ & $32.43(4.81)$ & $21.60(7.57)$ & 7.259 & $.027 *$ & .518 \\
\hline IEINTER & $23.67(8.50)$ & $23.71(3.63)$ & $16.20(6.18)$ & 4.125 & .127 & .294 \\
\hline IEADAP & $12.67(4.93)$ & $15.14(3.23)$ & $9.40(2.07)$ & 6.504 & $.039 *$ & .464 \\
\hline IEEST & $28.00(4.35)$ & $21.43(12.51)$ & $25.40(13.18)$ & .498 & .780 & .035 \\
\hline IEINTRA & $23.33(4.16)$ & $26.43(4.27)$ & $24.80(1.30)$ & 1.158 & .560 & .082 \\
\hline
\end{tabular}

Nota: (1) Media $=$ M, Desviación típica $=D T, \mathrm{H}$ de Kruskal Wallis $=\chi^{2}$, Optimismo $=$ OPT, Inteligencia emocional Atención = IEA, Claridad = IEAT, Reparación = IERE, Inteligencia emocional Interpersonal $=$ IEINTER, Adaptabilidad = IEADAP, Manejo del estrés $=$ IEEST, Intrapersonal = IEINTRA. $(2) *=p<.05 ; *=$ $p<.01$. (3) El tamaño del efecto estadístico está expresado con el valor Épsilon cuadrado $\left(E^{2}\right)$. 


\subsection{Estudio de regresión}

Con el propósito de analizar las variables que predicen la satisfacción vital (sWLS), considerada como variable criterio, se ha realizado un análisis de regresión lineal a través del método de pasos sucesivos. Se establecieron como variables predictoras cada una de las dimensiones de la inteligencia emocional del TMMs-24 (atención, claridad y reparación), EQi-C (interpersonal, adaptabilidad, manejo del estrés e intrapersonal) y el optimismo disposicional LOT-R (véase Tabla 5), asegurando, en primera instancia, que no existiese ningún problema de multicolinealidad (valores de tolerancia $<.20 ;$ FIV $>4.00$ ).

El resumen del modelo indica que solo queda incluida la dimensión de IE del EQi-C adaptabilidad (IEADAP); quedando excluidas las tres dimensiones del TMMS-24 (atención -IEA-, claridad -IEC-, reparación -IERE-), optimismo (OPT) y las tres dimensiones restantes del EQi-C (interpersonal -IEINTER-, manejo del estrés -IEEST- e intrapersonal -IEINTRA-).

\begin{tabular}{|c|c|c|c|c|c|c|c|}
\hline \multicolumn{8}{|c|}{$\begin{array}{c}\text { TABLA 5. Análisis de regresión lineal (pasos sucesivos), variable criterio: } \\
\text { satisfacción vital }\end{array}$} \\
\hline Variable criterio & $R$ & $R^{2}$ & $R^{2}$ corregida & $F$ & Variables predictoras & Beta & $T$ \\
\hline Satisfacción vital & .537 & .289 & .234 & 5.272 & & & \\
\hline & & & & & IEADAP & .705 & $2.296^{*}$ \\
\hline
\end{tabular}

Nota: (1) Optimismo = OPT, Inteligencia emocional Atención = IEA, Claridad = IEAT, Reparación = IERE, Inteligencia emocional Interpersonal = IEINTER, Adaptabilidad = IEADAP, Manejo del estrés = IEEST, Intrapersonal $=$ IEINTRA. $(2) *=p<.05$.

La dimensión incluida en la regresión explica un $28.9 \%$ de la varianza $(\mathrm{R}=.537$; $R^{2}$ corregida $\left.=.234 ; F=5.272 p<.05\right)$, existiendo un valor de $t$ significativo, siendo la mejor variable predictora de la satisfacción vital.

\section{Discusión}

Este trabajo parte de un análisis detallado de la IE, satisfacción vital y optimismo disposicional de las personas con DI que forman parte de un Centro de Día y Terapia Ocupacional $(n=20)$, centrando su desarrollo exclusivamente en los participantes con discapacidad leve o moderada $(n=15)$.

Los resultados encontrados evidenciaron que IE -EQi-C- (adaptabilidad) se asocia de forma significativa con una mayor satisfacción vital. Se observa una correlación positiva en ambos constructos, determinando que a mayor capacidad adaptativa de las personas con DI, mayor satisfacción con la vida, siguiendo los resultados obtenidos la misma tendencia que otros estudios (Cazalla-Luna y Molero, 2016; Cejudo et al., 2016; Extremera et al., 2011; Limonero, Tomás-Sábado, Fernández-Castro y Aradilla-Herrero, 2012; Sánchez-Álvarez, Extremera y Fernández-Berrocal, 2015). 
Otros trabajos sostienen las dificultades en las personas con Di para hacer frente de forma adaptativa a relaciones interpersonales (Medina-Gómez y Gil-Ibáñez, 2017).

Respecto a la relación establecida entre IE -TMMS-24- (atención y claridad), -EQiC- (intrapersonal) con optimismo disposicional, los hallazgos ponen de manifiesto una correlación significativa, coincidiendo con las evidencias halladas por Veloso-Besio et al. (2013). Esta asociación está justificada por el esfuerzo que hacen las personas por lograr sus objetivos cuando perciben que son alcanzables (Lench, 2011).

En función a las variables sociodemográficas género y edad, en relación con la satisfacción vital, no se han encontrado diferencias significativas, coincidiendo los hallazgos con otros estudios (Cazalla-Luna y Molero, 2016; Extremera, Durán y Rey, 2007; Vázquez et al., 2013). Debe apuntarse un mayor grado de satisfacción en hombres que en mujeres, dato que contradice estudios anteriores (Mestre, Samper, Frías y Tur, 2009) que informan una mayor empatía y predisposición a la satisfacción con la vida de la mujer, aunque deben considerarse los resultados con cautela, dado que los valores obtenidos corresponden a una muestra pequeña. En función de la edad, los datos informan de una mayor satisfacción vital en el intervalo de 26 a 39 años, en la línea de otros estudios que informan de que la satisfacción y bienestar subjetivo no decrecen con la edad (Wettstein, Schilling, Reidick y Wahl, 2015).

De igual forma, no se encuentran diferencias significativas entre las variables edad y género con el optimismo disposicional, coincidiendo con las evidencias resultantes de otras investigaciones (Cazalla-Luna y Molero, 2016; Extremera et al., 2007), siendo en nuestro estudio los hombres más optimistas que las mujeres, pudiendo estas evidencias estar condicionadas por factores culturales y sociales en el desarrollo de expectativas futuras (García-Naveira y Ruiz-Barquín, 2013). En nuestro caso, los participantes más jóvenes (<25 años) son los menos optimistas. Puede ser que las personas con DI con mayor edad y tiempo en el centro recuerden menos acontecimientos negativos que los más jóvenes (Charles et al., 2016) y, por tanto, tengan una visión más optimista en su futuro próximo.

En relación a los datos obtenidos entre IE y la variable sociodemográfica género, se constata que no se han encontrado diferencias significativas en el instrumento TMMS24, coincidiendo con otros estudios (Cazalla-Luna y Molero, 2016; De Haro y Castejón, 2014). Tampoco los resultados encontrados en EQi-C, en todas sus dimensiones, fueron significativos, al contrario de otros trabajos cuya tendencia sí muestra relación (Molero, Pantoja-Vallejo y Galiano-Carrión, 2017). Otros hallazgos sostienen que las personas con Di tienen especial dificultad para hacer frente de forma adaptativa a circunstancias adversas (Medina-Gómez y Gil-Ibáñez, 2017). Este estudio evidenció que los hombres presentan mayores niveles de IE, contradiciendo hallazgos anteriores (Cabello, Sorrel, Fernández-Pinto, Extremera y Fernández-Berrocal, 2016; Fernández-Berrocal et al., 2017), pero sin olvidar que trabajamos con un colectivo con DI, por lo que los resultados no podrían generalizarse a otros contextos.

Respecto a los resultados obtenidos en relación a satisfacción vital y optimismo disposicional respecto a la variable discapacidad se constata no encontrar diferencias significativas, siendo los valores encontrados en satisfacción vital más altos en las personas con grado de DI menor al 65\%, y más altas en optimismo para las personas con 
DI mayor al 65\%. Datos que contradicen estudios anteriores realizados con personas con discapacidad (Smedema et al., 2015), donde se apreciaba una clara relación entre satisfacción con la vida y discapacidad, superando planteamientos anteriores, donde la satisfacción con la vida y el bienestar subjetivo de las personas con DI disminuían por su condición (Veenhoven, 1994).

En relación a los datos obtenidos entre IE y la variable discapacidad, se constata no encontrar diferencias significativas en el instrumento TMMS-24. Sin embargo, en los resultados encontrados en EQi-C, su dimensión (interpersonal), sí resultó significativa.

En cuanto a las variables edad e IE sí encontramos diferencias significativas en TMMS-24 (claridad y reparación) y EQi-C (adaptabilidad), siendo el intervalo de 26 a 39 años el que puntuó más alto en las dos pruebas, por lo que se constata que aquellas personas con DI que presentan habilidades para adaptarse y manejar emociones propias y detectar las de los demás serán capaces de sobreponerse a situaciones adversas (Pulido y Herrera, 2016; Veloso-Besio et al., 2013). Los resultados obtenidos en relación a satisfacción vital y optimismo disposicional respecto a la variable edad no muestran diferencias significativas, siendo los valores encontrados en SWLS más altos en las personas menores de 25 años y más altas en LOT-R para las personas de entre 26 y 39 años.

Respecto al análisis de regresión, los resultados evidencian una sola variable de IE que predice una mayor satisfacción con la vida, dato a tener en consideración si tenemos en cuenta los instrumentos utilizados en IE en el modelo habilidad y modelo rasgo. El patrón confirma que la subescala IE EQi-C (adaptabilidad) entró en el modelo de regresión para explicar la satisfacción vital. El análisis de regresión múltiple realizado explicó un $28,9 \%$ de la variabilidad. Los resultados indican que las personas con DI que presentan respuestas adaptativas ante situaciones adversas tienen unos niveles más altos de satisfacción vital. Dato que contradice otras hipótesis donde se daban peores niveles en adaptación (Cejudo et al., 2016; Extremera et al., 2007; FernándezAbascal y Martín-Díaz, 2015).

Concluimos destacando la pertinencia de este estudio por varias cuestiones. En primer lugar, por lo novedoso en la temática e impacto que podría tener en estudios sobre la IE, satisfacción vital y optimismo disposicional en personas con DI, entendiendo su proyección para nuevos estudios con muestras más amplias y representativas, comparación entre diferentes centros y la realización de estudios longitudinales. En segundo lugar, aporta evidencias empíricas en la relación existente entre IE y satisfacción con la vida.

Dentro de las limitaciones del estudio, podemos señalar el tamaño reducido de la muestra y la dificultad en la obtención de datos, a pesar de ser personas con Di leve con capacidad limitada para la comprensión y la comunicación. Al tratarse de un grupo concreto, puede llevar a conclusiones poco representativas del colectivo general de personas con DI. Sin embargo, lo novedoso de nuestra propuesta pasa por la posibilidad de dar voz a la propia persona con DI, dato que en la mayoría de estudios no se tiene en consideración, dando relevancia a cada una de las evidencias descritas.

A pesar de ciertas limitaciones, nuestro estudio contribuye de manera significativa al campo de la IE, satisfacción vital y optimismo disposicional en colectivos de personas 
con DI, entendiendo su proyección para nuevos estudios con muestras más amplias y representativas, comparación entre diferentes centros y estudios longitudinales.

La veracidad y la relevancia de cada una de las evidencias descritas implicarán un mayor conocimiento en el bienestar personal del colectivo a estudio y servirá para mejorar la puesta en marcha de programas de IE en centros de atención a personas con DI.

\section{Referencias bibliográficas}

Alonso-Ferres, M., Berrocal de Luna, E. y Jiménez-Sánchez, M. (2018). Estudio sobre la inteligencia emocional y los factores contextuales en estudiantes de cuarto de educación primaria de la provincia de Granada. Revista de Investigación Educativa, 36 (1), 141-158. doi: http://dx.doi.org/10.6018/rie.36.1.281441.

Andrei, F. y Petrides, K. V. (2013). Trait emotional intelligence and somatic complaints with reference to positive and negative mood. Psibologija, 46, 5-15. doi: http://dx.doi.org/10.2298/ PSI1301005A.

Aradilla-Herrero, A., Tomás-Sábado, J. y Gómez-Benito, J. (2014). Perceived emotional intelligence in nursing: psychometric properties of the Trait Meta-Mood Scale. Journal of Clinical Nursing, 23, 955-966. doi: http://dx.doi.org/10.2298/PSI1301005A 10.1111/ jocn.12259.

Ato, M., López, J. J. y Benavente, A. (2013). Un sistema de clasificación de los diseños de investigación en psicología. Anales de Psicología, 29 (3), 1038-1059. doi: http://dx.doi. org/10.6018/ analesps.29.3.178511.

Augusto-Landa, J. M., Pulido-Martos, M. y López-Zafra, E. (2011). Does perceived emotional intelligence and optimism/pessimism predict psychological well-being? Journal of Happiness Studies, 12, 463-474. doi: http://dx.doi.org/10.1007/s10902-010-9209-7.

Avey, J. B., Luthans, F., Smith, R. M. y Palmer, N. F. (2010). Impact of positive psychological capital on employee well-being over time. Journal of Ocupational Health Psychology, 15 (1), 17-28. doi: http://dx.doi.org/10.1037/a0016998.

BAR-ON, R. (1997). The Emotional Quotient Inventory (EQ-i). A test of Emotional Intelligence. Toronto: Multi-Health Systems.

BAR-On, R. y PARker, J. D. (2000). The emotional quotient invetitorv: Youth version (EQ-i:YV). North Tonawanda, Nueva York: Multi-Health Systems.

Bassi, M., Bacher, G., Negri, L. y Delle Fave, A. (2013). The contribution of job happiness and job meaning to the well-being of workers from thriving and failing companies. Applied Research in Quality of Life, 8 (4), 427-448. doi: http://dx.doi.org/10.1007/ s11482-012-9202-x.

Bazzano, A., Wolfe, C., Zylowska, L., Wang, S., Schuster, E., Barrett, C. y Lehrer, D. (2015). Mindfulness based stress reduction (MBSR) for parents and caregivers of individuals with developmental disabilities: A community-based approach. Journal of Child and Family Studies, 24, 298-308. doi: http://dx.doi.org/10.1007/s10826-013-9836-9.

Bradburn, N. M. (1969). The structure of psychological well-being. Chicago: Aldine.

Cabello, R., Ruiz-Aranda, D. y Fernández-Berrocal, P. (2009). Docentes emocionalmente inteligentes. Revista Interuniversitaria de Formación del Profesorado, 13 (1), 41-49.

Cabello, R., Sorrel, M. A., Fernández-Pinto, I., Extremera, N. y Fernández-Berrocal, P. (2016). Age and gender differences in ability emotional intelligence in adults: A cross- 
ESTUDIO SOBRE INTELIGENCIA EMOCIONAL, SATISFACCIÓN VITAL Y OPTIMISMO DISPOSICIONAL EN UN CENTRO DE DÍA OCUPACIONAL DE PERSONAS CON DISCAPACIDAD INTELECTUAL ÓSCAR GAVÍN CHOCANO Y DAVID MOLERO

sectional study. Developmental Psychology, 52, 1486-1492. doi: http://dx.doi.org/10.1037/ dev0000191.

Carver, C. y Scheier, M. (2014). Dispositional optimism. Trends in Cognitive Sciences, 18 (6), 293-299. doi: http://dx.doi.org/10.1016/j.tics.2014.02.003.

Carver, C. S., Scheier, M. F. y Segerstrom, S. C. (2010). Optimism. Clinical Psychology Review, 30, 879-889. doi: http://dx.doi.org/10.1016/j.cpr.2010.01.006.

Cazalla-Luna, N. y Molero, D. (2016). Inteligencia emocional percibida, disposición al optimismo-pesimismo, satisfacción vital y personalidad de docentes en su formación inicial. Revista de Investigación Educativa, 34 (1), 241-258. doi: http://doi.org/10.6018/ rie.34.1.220701.

Cazalla-Luna, N. y Molero, D. (2018). Emociones, afectos, optimismo y satisfacción vital en la formación inicial del profesorado. Profesorado, Revista de Currículum y Formación del Profesorado, 22 (1), 215-233.

Cejudo, J., López, M. L. y Rubio, M. J. (2016). Inteligencia emocional y resiliencia: su influencia en la satisfacción con la vida de estudiantes universitarios. Anuario de Psicologia, 46, 51-57. doi: https://doi.org/10.1016/j.anpsic.2016.07.001.

Charles, S. T., Piazza, J. R., Mogle, J. A., Urban, E. J., Sliwinski, M. J. y Almeida, D. M. (2016). Age differences in emotional well-being vary by temporal recall. The Journals of Gerontology Series B: Psychological Sciences and Social Sciences, 71, 798-807. doi: http:// dx.doi.org/10.1093/geronb/gbv011.

Chavarria, M. P. y Barra, E. (2014). Satisfacción vital en adolescentes: Relación con autoeficacia y el apoyo social percibido. Terapia Psicológica, 32 (1), 41-46. doi: http://dx.doi. org/10.4067/S0728-4808201400010000.

CoHen, J. (1988). Statistical power analysis for the behavioral sciencies. Hillsdale, New York, EE. UU.: Erlbaum.

Contreras, D., Lillo, S. y Vera-Villarroel, P. (2015). Subjective sexual well-being in chilean adults: Evaluation of a predictive model. Journal of Sex and Marital Therapy, 42 (4), 338-52. doi: http://dx.doi.org/10.1080/0092623X.2015.1053018.

De Haro, J. M. y Castejón, J. L. (2014). Perceived emotional intelligence, general intelligence and early professional success: predictive and incremental validity. Anales de Psicología, 30 (2), 490-498. doi: http://dx.doi.org/10.6018/analesps.30.2.154621.

Diener, E. D., Emmons, R. A., Larsen, R. J. y Griffin, S. (1985). The satisfaction with life scale. Journal of Personality Assessment, 49, 71-75.

Diener, E. D., Oishi, S. y RYAn, K. (2013). Universal and cultural differences in the causes and structure of "happiness" - A multilevel review. En C. KeYEs (Ed.), Mental wellbeing: International contributions to the study of positive mental healths (pp. 153-176). Dordrecht, Netherlands: Springer.

Extremera, N., Durán, A. y Rey, L. (2005). La inteligencia emocional percibida y su influencia sobre la satisfacción vital, la felicidad subjetiva y el engagement en trabajadores de centros para personas con discapacidad intelectual. Ansiedad y Estrés, 11 (1), 63-73.

Extremera, N., Durán, A. y Rey, L. (2007). Perceived emotional intelligence and dispositional optimism-pessimism: Analyzing their role in predicting psychological adjustment among adolescents. Personality and Individual Differences, 42, 1069-1079. doi: http://dx.doi. org/10.1016/j.paid.2006.09.014.

Extremera, N. y Fernández-Berrocal, P. (2006). Emotional intelligence as predictor of the mental, social, and physical health in university students. Spanish Journal of Psychology, 9, 45-51. doi: http://dx.doi.org/10.1017/S1138741600005965.

(C) Ediciones Universidad de Salamanca / CC BY-NC-ND

Siglo Cero, vol. 50 (3), n. ${ }^{\circ}$ 271, 2019, julio-septiembre, pp. 47-65 
ESTUDIO SOBRE INTELIGENCIA EMOCIONAL, SATISFACCIÓN VITAL Y OPTIMISMO DISPOSICIONAL EN UN CENTRO DE DÍA OCUPACIONAL DE PERSONAS CON DISCAPACIDAD INTELECTUAL ÓSCAR GAVÍN CHOCANO Y DAVID MOLERO

Extremera, N., Salguero, J. M. y Fernández-Berrocal, P. (2011). Trait meta-mood and subjective happiness: A 7-week prospective study. Journal of Happiness Studies, 12, 509517. doi: http://dx.doi.org/10.1007/s10902-010-9233-7.

Fernandes de Araújo, L., Teva, I. y Bermúdez, M. P. (2015). Resiliencia en adultos: una revisión teórica. Terapia Psicológica, 33, 257-276. doi: http://dx.doi.org/10.4067/S071848082015000300009.

Fernández-Abascal, E. y Martín-Díaz, M. D. (2015). Dimensions of emotional intelligence related to physical and mental health and to health behaviors. Frontiers in Psycology, 6, 317. doi: http://dx.doi.org/10.3389/fpsyg.2015.00317.

Fernández-Berrocal, P. Extremera, N. y Ramos, N. (2004). Validity and reliability of the Spanish modified version of the Trait Meta-Mood Scale. Psychological Reports, 94, 751-755. doi: http://dx.doi.org/10.2466/pro.94.3.751-755.

Fernández-Berrocal, P. y Ruiz-Aranda, D. (2008). La inteligencia emocional en la educación. Education and Psychology, 6 (15), 421-436.

Fernández-Berrocal, P., Ruiz-Aranda, D., Salguero, J. M., Palomera, R. y Extremera, N. (2017). La relación del Test de Inteligencia Emocional de la Fundación Botín (TIEFBA) con el ajuste personal y escolar de adolescentes españoles. Santander: Fundación Botín. doi: http://dx.doi.org/10.1016/j.psicod.2017.07.001.

García-Naveira, A. y Ruiz-Barquín, R. (2013). La personalidad del deportista: una revisión teórica desde la perspectiva de rasgos. Revista Internacional de Medicina y Ciencias de la Actividad Física y el Deporte, 13 (51), 627-645.

Gázquez-Linares, J., Pérez-Fuentes, M., Mercader-Rubio, I. e Inglés-Saura, C. (2014). Repercusión del optimismo y de los Cinco Grandes factores de la personalidad sobre la salud de personas mayores. Universitas Psychologica, 13 (3), 995-1004. doi: http://dx.doi. org/10.11144/Javeriana.UPSY13-3.rocg.

lara, F. J. P., Carranque, G., Oehling, H., Hernández, J. M. y Oliva, H. (2014). Psychological modulation in patients surgically intervened for gastroesophageal reflux disease. Diseases of the Esophagus, 27, 538-546.

Latorre, A., Del Rincón, D. y Arnal, J. (2003). Bases metodológicas de la investigación educativa. Barcelona: Ediciones Experiencia.

LeNCH, H. (2011). Personality and health outcomes: Making positive expectations a reality. Journal of Happiness Studies, 12 (3), 493-507. doi: http://dx.doi. org/10.1007/s10902-0109212-z.

Liberalesso, A. (2002). Bienestar subjetivo en la vida adulta y la vejez: hacia una psicología positiva en América Latina. Revista Latinoamericana de Psicología, 34, (1-2), 55-74.

Limonero, J. T., Tomás-Sábado, J., Fernández-Castro, J. y Aradilla-Herrero, A. (2012). Estrategias de afrontamiento resilientes y regulación emocional: predictores de satisfacción con la vida. Behavioral Psychology/Psicología Conductual, 20 (1), 183-189.

Londoño-Pérez, C., Velasco-Salamanca, M., Alejo-Castañeda, I., Botero-Soto, P. y VaNEGAS, I. (2014). What makes us optimistic? Psychosocial factors as predictors of dispositional optimism in young people. Terapia Psicológica, 32 (1), 41-46.

López-Cassá, E., Pérez-Escoda, N. y Alegre, A. (2018). Competencia emocional, satisfacción en contextos específicos y satisfacción con la vida en la adolescencia. Revista de Investigación Educativa, 36 (1), 57-73. doi: http://dx.doi.org/10.6018/rie.36.1.273131.

López-Zafra, E., Pulido-Martos, M. y Berrios, P. (2014). eqi Versión Corta (eqi-C). Adaptación y validación al español del EQ-i en universitarios. Boletín de Psicología, 110, 21-36. 
ESTUDIO SOBRE INTELIGENCIA EMOCIONAL, SATISFACCIÓN VITAL Y OPTIMISMO DISPOSICIONAL EN UN CENTRO DE DÍA OCUPACIONAL DE PERSONAS CON DISCAPACIDAD INTELECTUAL ÓSCAR GAVÍN CHOCANO Y DAVID MOLERO

MacCann, C., Joseph, D. L., Newman, D. A. y Roberts, R. D. (2014). Emotional intelligence is a second-stratum factor of intelligence: Evidence from hierarchical and bifactor models. Emotion, 14, 358-374. doi: http://dx.doi.org/10.1037/a0034755.

Manor-Binyamini, I. y Nator, M. (2016). Parental coping with adolescent developmental disabilities in terms of stress, sense of coherence and hope within the Druze community of Israel. Research in Developmental Disabilities, 55, 358-367. doi: http://dx.doi.org/10.1016/j. ridd.2016.05.003.

Marrero, R. y Caballeira, M. (2010). El papel del optimismo y el apoyo social en el bienestar subjetivo. Salud Mental, 33, 39-46.

Mavroveli, S., Petrides, K. V., Sangareau, Y. y Furnham, A. (2009). Relating trait emotional intelligence to objective socioemotional outcomes in childhood. British Journal of Educational Psychology, 79, 259-272.

Mayer, J. D., Roberts, R. D. y Barsade, S. G. (2008). Human abilities: Emotional Intelligence. Anual Review of Psychology, 59, 507-536. doi: https://doi.org/10.1146/annurev. psych.59.103006.093646.

Mayer, J. D., Salovey, P. y Caruso, D. (2000). Models of emotional intelligence. En R. J. SterNBERG (Ed.), Handbook of Intelligence (pp. 396-420). New York: Cambridge.

Medina, B. y GiL, R. (2017). Estrés y estrategias de afrontamiento en personas con discapacidad intelectual: revisión sistemática. Ansiedad y Estrés, 23, 38-44. doi: http://dx.doi. org/10.1016/j.anyes.2017.05.001.

Mestre, V., Samper, P., Frías, D. y Tur, A. (2009). Are women more empathetic than men? A longitudinal study in adolescence. The Spanish Journal of Psychology, 12 (1), 76-83. doi: https://doi.org/10.1017/S1138741600001499.

Molero, D., Pantoja, A. y Galiano, M. (2017). Inteligencia emocional rasgo en la formación inicial del profesorado. Contextos Educativos: Revista de Educación, 20, 43-56. doi: http:// dx.doi.org/10.18172/con.2993.

Munkholm, K., Vinberg, M., Berk, M. y Kessing, L. V. (2012). State-related alterations of gene expression in bipolar disorder: a systematic review. Bipolar Disorders, 14, 684-696. doi: https://doi.org/10.1111/bdi.12005.

NovoA, C. y BARra, E. (2015). Influencia del apoyo social percibido y los factores de personalidad en la satisfacción vital de estudiantes universitarios. Terapia Psicológica, 33, 239-245.

Pedrosa, I., Celis-Atenas, K., Suárez-Álvarez, J., García-Cueto, E. y Muñiz, J. (2015). Cuestionario para la evaluación del optimismo: Fiabilidad y evidencias de validez. Terapia Psicológica, 33 (2), 127-138.

Petrides, K. V. y Furnham, A. (2001). Trait emotional intelligence: Psychometric investigation with reference to established trait taxonomies. European Journal of Personality, 15, 425-448. doi: http://dx.doi.org/10.1002/per.416.

Petrides, K. V., Pérez-González, J. C. y Furnham, A. (2007). On the criterion and incremental validity of trait emotional intelligence. Cognition and Emotion, 21, 26-55. doi: https:// doi.org/10.1080/02699930601038912.

Pulido, F. y Herrera, F. (2018). Relaciones entre rendimiento e inteligencia emocional en secundaria. Tendencias Pedagógicas, 31, 165-185.

Remor, E., Amorós, M. y Carrobles, J. A. (2006). El optimismo y la experiencia de ira en relación con el malestar físico. Anales de Psicología, 22 (1), 37-44.

Rodríguez, M. J. (2015). Representación social de la noción de felicidad en estudiantes y profesionales de educación y salud de Chile e Italia. Universitas Psychologica, 14 (1). doi: http://dx.doi.org/10.11144/Javeriana.upsy 14-1.rsnf.

(C) Ediciones Universidad de Salamanca / CC BY-NC-ND

Siglo Cero, vol. 50 (3), n. ${ }^{\circ}$ 271, 2019, julio-septiembre, pp. 47-65

$$
-63-
$$


ESTUDIO SOBRE INTELIGENCIA EMOCIONAL, SATISFACCIÓN VITAL Y OPTIMISMO DISPOSICIONAL EN UN CENTRO DE DÍA OCUPACIONAL DE PERSONAS CON DISCAPACIDAD INTELECTUAL ÓSCAR GAVÍN CHOCANO Y DAVID MOLERO

Rojas, M. y Veenhoven, R. (2013). Contentment and affect in the estimation of happiness. Social Indicators Research, 110, 415-431. doi: http://dx.doi.org/10.1007/s11205-011-9952-0.

Salovey, P. y Mayer, J. D. (1990). Emotional Intelligence. Imagination, Cognition y Personality, 9 (3), 185-211. doi: https://doi.org/10.2190/DUGG-P24E-52WK-6CDG.

Salovey, P., Mayer, J. D., Goldman, S. L., Turvey, C. y Palfai, T. P. (1995). Emotional attention, clarity and repair: exploring emotional intelligence using the Trait Meta-Mood Scale. En J. W. Pennebaker (Ed.), Emotion, disclosure and health (pp. 125-154). Washington: American Psychological Association. doi: http://dx.doi.org/10.1037/10182-006.

Sánchez-Álvarez, N., Extremera, N. y Fernández-Berrocal, P. (2015). The relation on betwen emotional intelligence and subjective well-being: A meta-analytic investigation. The Journal of Positive Psychology, 11 (3), 276-285. doi: http://dx.doi.org/10.1080/174397 60.2015.1058968.

Scheier, M. y Carver, C. (1985). Optimism, coping, and health: Assessment and implications of generalized outcome expectancies. Health Psychology, 4, 219-247.

Scheier, M., Carver, C. y Bridges, M. (1994). Distinguishing optimism from neuroticiscm (and trait anxiety, self-mastery, and self-esteem): A reevaluation of the Life Orientation Test. Journal of Personality and Social Psychology, 67 (6), 1063-1078.

Schimmack, U. (2007). The structure of subjective wellbeing. En R. LARSEN y M. EID (Eds.), The science of subjective well-being (pp. 97-123). New York: Guilford.

ŞIMSsEK, O. F. y Koydemir, S. (2013). Linking metatraits of the big five to well-and ill-being: Do basic psychological needs matter? Social Indicators Research, 112, 221-238. doi: http:// dx.doi.org/10.1007/s11205-012-0049-1.

Smedema, S., Chan, F., Yaghmaian, R., Cardoso, E., Muller, V., Keegan, J., Dutta, A. y EBener, D. (2015). The relationship of core self-evaluations and life satisfaction in college students with disabilities: evaluation of a mediator model. Journal of Postsecondary Education and Disability, 28, 341-358.

Thompson, B. L., Waltz, J., Croyle, K. y Pepper, A. C. (2007). Trait meta-mood and affect as predictors of somatic symptoms and life satisfaction. Personality and Individual Differences, 43, 1786-1795. doi: http://dx.doi.org/10.1016/j.paid.2007.05.017.

Tolegenova, A. A., Kustubayeva, A. M. y Matthews, G. (2014). Trait meta-mood, gender and EEG response during emotion-regulation. Personality and Individual Differences, 65, 75-80. doi: http://dx.doi.org/10.1016/j.paid.2014.01.028.

Veenhoven, R. (1994). El estudio de la satisfacción con la vida. Intervención Psicosocial, 3, 87-116.

Veloso-Besio, C., Cuadra-Peralta, A., Antezana-Saguez, I., Avendaño-Robledo, R. y Fuentes-SOTO, L. (2013). Relación entre inteligencia emocional, satisfacción vital, felicidad subjetiva y resiliencia en funcionarios de Educación Especial. Estudios Pedagógicos, 39 (2), 355-366. doi: http://dx.doi.org/10.4067/s0718-07052013000200022.

Vera-Villarroel, P., PÁvez, P. y Silva, J. (2012). El rol predisponente del optimismo: hacia un modelo etiológico del bienestar. Terapia Psicológica, 30 (2), 77-84. doi: http://dx.doi. org/10.4067/S0718-48082012000200008.

Von Humboldt, S. y Leal, I. (2014). ¿Qué incluye en el bienestar subjetivo de los adultos mayores? Una revisión sistemática de la literatura. Revista Argentina de Clínica Psicológica, 23 (3), 219-230.

Wasserstein, R. L. y Lazar, N. A. (2016). The asa's Statement on p-Values: Context, process, and purpose. The American Statistician, 70 (2), 129-133. doi: https://doi.org/10.1080/0003 1305.2016.1154108.

(C) Ediciones Universidad de Salamanca / CC BY-NC-ND

Siglo Cero, vol. 50 (3), n. ${ }^{\circ}$ 271, 2019, julio-septiembre, pp. 47-65

$$
-64-
$$


ESTUDIO SOBRE INTELIGENCIA EMOCIONAL, SATISFACCIÓN VITAL Y OPTIMISMO DISPOSICIONAL

EN UN CENTRO DE DÍA OCUPACIONAL DE PERSONAS CON DISCAPACIDAD INTELECTUAL

ÓSCAR GAVÍN CHOCANO Y DAVID MOLERO

Wettstein, M., Schilling, O. K., Reidick, O. y Wahl, H. W. (2015). Four-year stability, change, and multidirectionality of well-being in very-old age. Psychology and Aging, 30 (3), 500-516. doi: http://dx.doi.org/10.1037/pag0000037.

Xu, J. y RoberTs, R. (2010). The power of positive emotions: It's a matter of life or death-subjective well-being and longevity over 28 years in a general population. Health Psychology, 29, 9-19. doi: http://dx.doi.org/10.1037/a0016767. 


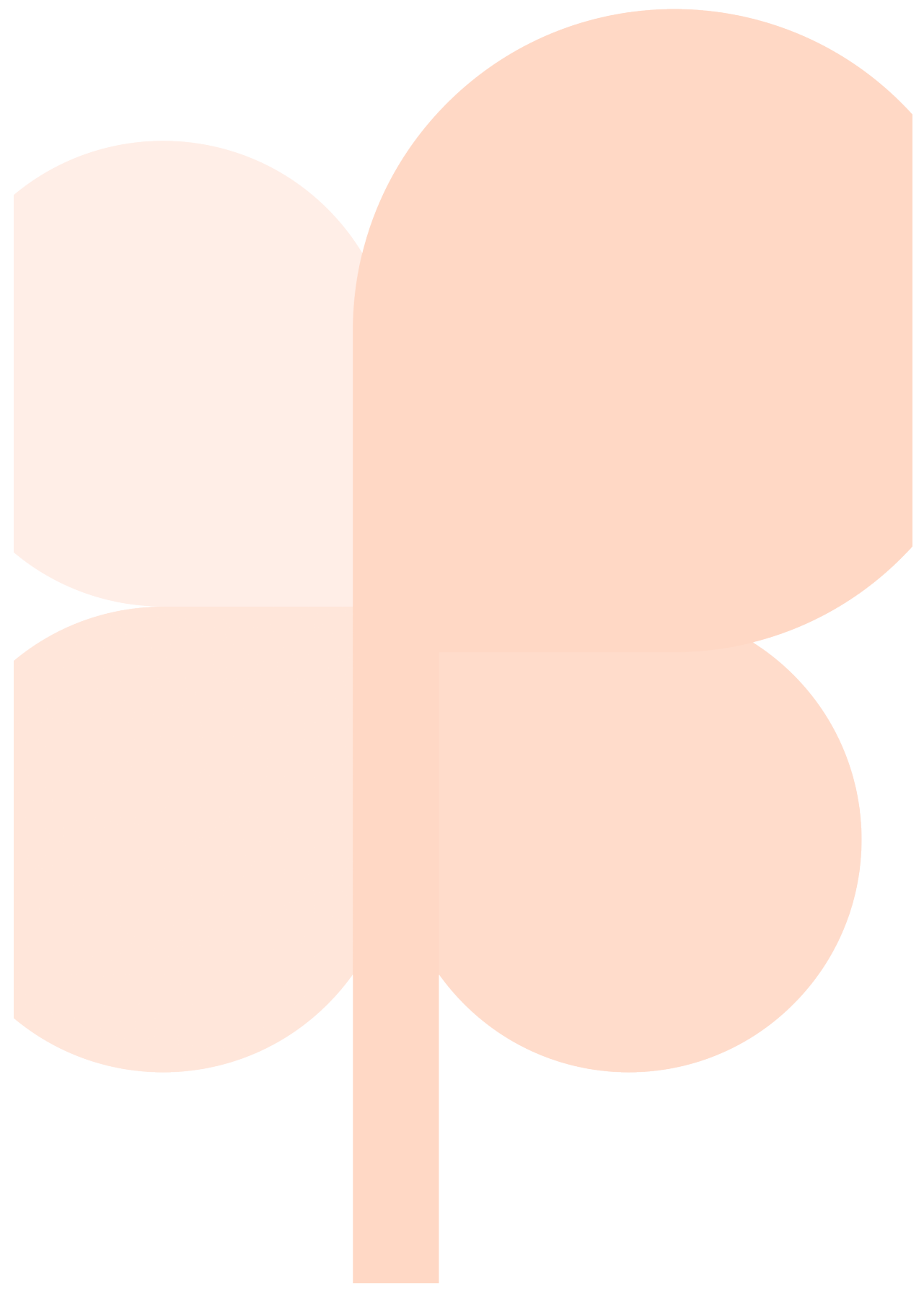

